\title{
Resistencias mecánicas del cemento Estudio de la precisión de su medida
}

CESAR DEL OLMO RODRIGUEZ (*) IETCC/CSIC

\author{
$R E S U M E N$ \\ El presente trabajo contiene un estudio efectuado para \\ determinar la precisión del método normalizado \\ de ensayo que se aplica para la medida de \\ las resistencias mecánicas del cemento, o en otras \\ palabras, para conocer la dispersion de valores que \\ cabe esperar, tanto al repetir con un material \\ cl ensayo en un mismo laboratorio (repetibilidad), \\ como al efectuar el ensayo en laboratorios diferentes. \\ (reproducibilidad) \\ Con la finalidad arriba apuntada se ha realizado \\ el cinsa!ro colectivo culos resultados aqui se reseñan, \\ efectuado en el marco del Grupo de Trabajo 1 "Ensavos \\ Físicos y' Mecánicos" de la Comisión Técnica \\ " 80 Cementos. Cales del IRANOR, en el que han \\ tomado parte 10 laboratorios afectos al $G T$
}

\author{
$S U M M A R Y$ \\ The present work contains a study effected in order \\ to determine the precision of the standardized test method \\ which is applied for the measurement of the mechanical \\ resistences of cement or; in other words, to know the \\ dispersion of values which may be expected, both in \\ repeating with a matcrial the test in the same laboratory \\ (repetition), and carrying out the test in different \\ laboratories (reproduction) \\ With the above mentioned object the collective test \\ has been carried out whose results are included here, \\ carried out within the fiamework of the Work Group \\ Phisical and Mechanical Tests of the Technical \\ Commission No 80 Cements and limes of IRANOR, in \\ which 10 laboratories of ( $T$ took part
}

\section{INTRODUCCION}

La Comisión Técnica n. ${ }^{\circ} 80$ "Cementos y Cales" del Instituto Español de Normalización (IRANOR) ha recibido el encargo de actualizar y adaptar las normas españolas sobre estos materiales a las correspondientes europeas (CEN), con la finalidad de que en un futuro las normas por las que se rigen estos materiales en España, en cuanto a especificaciones, métodos de ensayo, etc., tengan un carácter más universal, favoreciéndose con ello el intercambio comercial (téngase en cuenta que España es uno de los primeros paises exportadores de cemento mundiales) y facilitando además, en el campo de la normalización, la integración futura de nuestro pais en el mercado común.

Para mejor realizar la labor anteriormente mencionada, dentro de la Comisión Técnica $n .{ }^{\circ} 80$ se han creado cuatro Grupos de Trabajo (G.T.) que estudian cada uno de los temas en que se ha dividido dicha labor, asi por ejemplo, el G.T. 1 es el encargado de los ensayos Físicos y Mecánicos.

Este grupo ha iniciado su trabajo con el estudio del método de ensayo para determinar las resistencias mecánicas del cemento, habiendo dado por finalizada la redacción de la norma UNE 80101 correspondiente, que ha sido remitida al IRANOR para su publicación como Proyecto de Norma UNE, iniciándose con ello el periodo de encuesta pública antes de la adopción definitiva.

$\left({ }^{*}\right)$ Doctor en Ciencias Quimicas. Secretario del G.T. 1 de la Comisión Técnica 80 del IRANOR. 
En la pasada fase de estudio por el G.T. del proyecto de norma mencionada (UNE 80 101), y con la finalidad de estimar la precisión del método de medida de esta caracteristica del cemento, se efectuó el ensayo colectivo descrito en este trabajo, que se difunde por acuerdo del G.T.

\section{CAUSAS GENERALES DE DISPERSION}

La medida de una característica en un material de construcción es, por lo general, el resultado de una serie de operaciones complejas realizadas por uno o varios operadores con ayuda de un instrumental diverso.

Como consecuencia del proceso anterior, el valor obtenido en la medida puede venir afectado por dispersiones, cuya magnitud depende de varios factores que no siempre actúan en el mismo sentido de elevar o reducir el resultado del ensayo. Estos factores pueden ser sistemáticos o accidentales.

Entre estos factores aludidos cabe mencionar los siguientes:

1. que el método de medida adolezca de imprecisiones, por lo que respecta a:

1.1. las operaciones que comprende el ensayo;

1.2. las caracteristicas de los aparatos utilizados en el mismo, en nuestro caso: amasadora, compactadora, moldes, prensas, etc.;

2. que los aparatos estén, en mayor o menor medida, fuera de norma, bien por deficiencias de fabricación, o de conservación (p. ejem. por no sustituir los elementos desgastados, algunos de los cuales tienen una influencia decisiva en el resultado del ensayo $\left.{ }^{*}\right)$; y/o también

que las instalaciones (cámaras de conservación y curado, laboratorios, etc.) no estén debidamente acondicionadas,

que el material auxiliar utilizado (agua de amasado, arena normalizada, etc.) no reuna las características exigidas;

3. que el operador cometa algún error, bien en la interpretación del método aplicado, o en la realización de las operaciones de que consta el ensayo, etc.;

4. que el material ensayado no tenga una constancia de calidad de unas partidas a otras es también una fuente de dispersiones $(* *)$; por útimo

5. que se produzcan durante el ensayo fallos erráticos;

5.1. en los aparatos;

5.2. en las instalaciones (p. ejem., como consecuencia de un corte en el suministro eléctrico del sistema de regulación automático de temperatura, la cámara de curado puede sufrir una reducción transitoria de temperaturas que puede originar una caída de resistencias en el cemento);

5.3. en el material auxiliar (p. ejem. el agua de amasado o la arena normalizada contiene agentes que impiden una buena adherencia entre los componenetes del mortero);

5.4. en el operador (faltas de atención esporádicas, etc.).

(*) Este es el caso de los platos de las prensas donde se colocan las probetas para el ensayo de compresion.

(**) En el ensayo colectivo que aqui se expone. esta causa de dispersion ha sido especialmente obviada mediante una homogencizacion muy cuidada del material ensayado. 


\section{ENSAYO COLECTIVO}

El ensayo colectivo interlaboratorios realizado con la finalidad anteriormente expresada, ha consistido en la determinación de las resistencias mecánicas a compresión del cemento, únicas para las que la norma CEN $\left(^{*}\right)$ correspondiente establece especificaciones (por esta razón, los resultados a flexotracción no han sido tomados en consideración en este caso).

La estimación de la precisión del método de ensayo se ha realizado a partir de los resultados obtenidos por 10 operadores diferentes, trabajando con equipos distintos en laboratorios diferentes con muestras, idénticas en lo posible, de dos materiales (cementos).

Para la realización del ensayo cada laboratorio ha hecho uso del utillaje (amasadora, compactadora, moldes, prensa, etc.) normalizado de que disponía -que corresponde al que se describe en el Pliego español RC-75 (**) vigente-, pero introduciendo en la técnica operatoria del ensayo las pequeñas modificaciones que establece la norma CEN correspondiente. Estas modificaciones apuntadas no son, en la mayoría de los casos, más que puntualizaciones acerca de algunas operaciones en cuya ejecución en el Pliego RC-75 se deja un cierto margen de libertad.

Los cementos ensayados han sido dos comerciales de los tipos y categorias siguientes:

- PA-350, y

- P-450 ARI

En el ensayo para la confección de las probetas se ha usado arena normalizada procedente de la misma partida.

Las edades de conservación a las que se ha efectuado el ensayo han sido las habituales de:

- 7 dias, $\mathrm{y}$

- 28 dias.

Para el ensayo colectivo de referencia, cada laboratorio ha fabricado, conservado bajo agua y roto en sus prensas, 3 series de probetas $(* * *)$ de tres probetas por serie, por cada cemento ensayado y por edad de conservación, por lo que el número de valores individuales de resistencias de compresión obtenidos en cada caso es de 18, dado que cada probeta ensayada proporciona dos valores.

Dos particularidades han sido adoptadas en el ensayo, en primer lugar, que cada serie de probetas utilizadas en la medida de una característica ha sido fabricada en días distintos y sucesivos; y también, siguiendo la técnica operatoria del método CEN, que, de las 6 probetas fabricadas cada dia, las situadas en el primer molde fabricado en las dos posiciones laterales, junto con la probeta central del segundo molde, forman el conjunto de tres probetas que se destina para realizar, en la fecha oportuna, el ensayo de resistencias mecánicas a la edad de 7 dias, destinándose las probetas restantes (es decir, la probeta central del primer molde y las laterales del segundo) al ensayo de 28 días.

En el ensayo colectivo han participado un total de 10 laboratorios, todos ellos con experiencia

(*) Estrictamente, la norma CEN a la que se alude repetidamente en este trabajo, corresponde al proyecto de norma pr EN 196-1 que será sometido al voto final de los paises miembros del CEN cl dia 31-I-84.

(**) Pliego de Prescripciones Ténicas Generales para la Recepción de Cemento RC-75 (B.O.F. n." 206 de 28 de agosto de 1975).

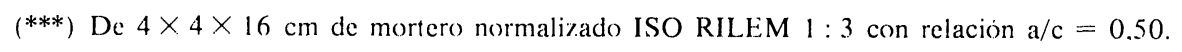


en la realización de este tipo de ensayos. Estos laboratorios pertenecen a los tres colectivos siguientes: organismos oficiales, industria cementera y laboratorios homologados.

Los resultados obtenidos figuran en los Cuadros de valores III al $\mathrm{X}$ que se incluyen al final del trabajo. Estos cuadros contienen los valores de las resistencias mecánicas a compresión, expresadas en $\mathrm{Kp} / \mathrm{cm}^{2}$, y están divididos en la forma siguiente:

$$
\begin{aligned}
& \text { Cuadros } \\
& \hline \text { III y VII } \\
& \text { IV y VIII } \\
& \text { V y IX } \\
& \text { VI y X }
\end{aligned}
$$

$\frac{\text { Cemento }}{\text { PA-350 a } 7 \text { días }}$
PA-350 a 28 días
P-450 ARI a 7 dias
P-450 ARI a 28 dias

En los cuatro primeros Cuadros de valores indicados se facilitan todos los valores individuales obtenidos por los 10 laboratorios participantes en las tres series de probetas que ha comprendido el ensayo; a partir de estos valores se han calculado los parámetros estadísticos que se mencionan en el apartado siguiente.

En los restantes Cuadros de valores mencionados se incluyen igualmente los resultados de las tres series de probetas (obtenidos estos resultados como media de 6 valores individuales, tal como indica el método normalizado de ensayo), a partir de estos resultados se han calculado, del mismo modo, los parámetros estadísticos que se describen en el apartado siguiente.

\section{ESTUDIO ESTADISTICO}

El análisis estadístico de los resultados del ensayo colectivo ha sido efectuado por varios métodos, uno de ellos es el que se describe en este trabajo y corresponde al procedimiento operatorio de la norma ASTM C $802\left({ }^{*}\right)$, que establece una mecánica operatoria para evaluar la precisión de un método de ensayo que se aplica para determinar cualquier caracteristica de un material de construcción, a través de un programa colectivo de ensayos como el que nos ocupa.

El proceso de datos del ensayo colectivo ha sido efectuado por la Sección de Cálculo del IET haciendo uso de un programa de ordenador preparado para este fin.

Los parámetros determinados en el estudio estadístico efectuado han sido calculados de dos formas distintas.

- en primer lugar, a partir de los 18 valores individuales de que constan las 3 series de ensayos efectuados, para cada cemento considerado y edad de conservación, por los 10 laboratorios participantes [modalidad (1) de cálculo]; y

- en segundo lugar, a partir de los 3 resultados obtenidos en las 3 series de ensayos anteriores [modalidad (2)].

Estos parámetros estadísticos están incluidos en los 8 Cuadros de valores anteriormente mencionados.

Los parámetros estadisticos calculados han sido los siguientes:

1) Para cada laboratorio (i):

- el valor medio de la serie $\left(\bar{x}_{\mathrm{i}}\right)$.

(*) American Socicty for Testing and Materials. 
- La varianza de la serie $\left(s_{\mathrm{i}}^{2}\right)$, que representa una medida (un indice) de la dispersión de los valores que comprende la serie $(*)$.

- La desviación del valor medio de la serie $\left(\bar{x}_{\mathrm{i}}\right)$ con relación al valor medio global $\left(\bar{x}_{\mathrm{A}}\right)$ de todos los laboratorios, expresada en tanto por ciento.

2) Para el conjunto (A) de todos los laboratorios participantes:

- El valor medio global del conjunto de resultados de todos los laboratorios $\left(\bar{x}_{\mathrm{A}}\right)$.

- La varianza del conjunto de laboratorios $\left(s_{\AA}^{2}\right)$.

- La desviación típica del conjunto de laboratorios $\left(\sigma_{\mathrm{A}}\right)$, como una medida de la repetibilidad de resultados de un laboratorio promedio.

- El coeficiente de variación, en tanto por ciento $\left(V_{\mathrm{A}}, \%\right)$ del conjunto de laboratorios, como medida de otro aspecto de la repetibilidad de resultados en un laboratorio promedio.

- La desviación típica entre laboratorio $\left(\sigma_{\mathrm{EL}}\right)$, como media de la reproducibilidad de resultados entre laboratorios.

- El coeficiente de variación entre laboratorios $\left(V_{\mathrm{FLL}}, \%\right)$, como medida de otro aspecto de la reproducibilidad de resultados entre laboratorios.

En los Cuadros de valores (III al X) varias veces mencionados se incluyen integramente todos los valores obtenidos en el Ensayo Colectivo, sin que se haya procedido a eliminar aquellas series que se encuentran afectadas por una dispersión -medida por la varianza $\left(s_{i}^{2}\right)$ - superior a la que admite el método estadístico aplicado. Por la razón expuesta, el presente estudio ha sido efectuado considerando el caso más desfavorable; de haberse eliminado las series erráticas, las dispersiones $\left(s_{\AA}^{2}\right)$ que se habrian obtenido serian inferiores a las indicadas $(* *)$.

No obstante, cabe señalar que los resultados de las series incluidas en los Cuadros de valores VIII y X, que han servido para el cálculo de los valores considerados en la norma CEN de referencia [resistencias mecánicas a compresión a 28 días, calculadas según (2)], -a que se refieren: los Cuadros I y II, así como la Fig. 1- no están afectados por dispersiones por encima del nivel tolerado por el método estadístico aplicado, por lo que estos resultados son correctos desde este punto de vista (estadístico).

\subsection{Cálculo de los parámetros estadísticos}

Las expresiones matemáticas aplicadas para el cálculo de los parámetros estadisticos anteriores se indican a continuación: sin embargo se renuncia a la descripción de los aspectos teóricos que conducen a dichas expresiones, por quedar fuera de la finalidad de este trabajo $(* * *)$.

Estas expresiones son las siguientes:

1) Para la serie formada por cada laboratorio (i) al efectuar la determinación de una caracteristica.

(*) Cuanto mayor sea el valor de $s_{\mathrm{i}}^{2}$, mayores dispersiones se han producido en el ensayo.

(**) El método estadistico aplicado establece unas limitaciones para los valores que pueden alcanzar las varianzas de las series. Estas limitaciones se calculan como relación: a) entre las varianzas $\left(s_{\mathrm{i}}^{2}\right)$ máximas y minimas: y b) entre las varianzas máxima y media $\left(s_{\mathrm{A}}^{2}\right)$. Asi por ej. para el caso de 10 laboratorios y de 3 series de valores. los valores máximos admitidos que pueden alcanzar las relaciones (o cocientes) entre las mágnitudes indicadas, son respectivamente de: 550 y 5 .

$\left(^{* * *}\right)$ Mayor información sobre el tema puede obtenerse no obstante consultando la bibliografia incluida al final del trabajo. 
Valor medio $\left(\bar{x}_{\mathrm{i}}\right)$ de la serie de valores o resultados obtenidos.

$$
\bar{x}_{\mathrm{i}}=\sum x_{\mathrm{i}} / n
$$

o suma de los valores o resultados obtenidos dividido por el número $(n)$ de ensayos efectuados.

Varianza de la serie $\left(s_{\mathrm{i}}^{2}\right)$ de valores obtenidos por un laboratorio

$$
s_{\mathrm{i}}^{2}=\left(\sum x_{\mathrm{i}}^{2}-n \bar{x}_{\mathrm{i}}^{2}\right) /(n-1)
$$

o suma de los cuadrados de los $n$ resultados del ensayo repetido por un laboratorio $(i)$ menos $n$ veces el cuadrado del valor medi $\left(\bar{x}_{i}\right)^{-}$) obtenido por este laboratorio, dividido por el número $(n)$ de ensayos efectuados menos 1 .

Desviación del valor medio en tanto por ciento (desviación media, \%) de la media $\left(\bar{x}_{\mathrm{i}}\right)$ con relación al valor medio global $\left(\bar{x}_{\mathrm{A}}\right)$ del conjunto, expresado en tanto por ciento

$$
\text { desviación media, } \%=\frac{\bar{x}_{\mathrm{A}}-\bar{x}_{\mathrm{i}}}{\bar{x}_{\mathrm{A}}} 100
$$

2) Para la serie (A) formada por el conjunto de los resultados obtenidos por los 10 laboratorios al efectuar la medida de una misma característica.

Valor medio global $\left(\bar{x}_{\mathrm{A}}\right)$ del conjunto de laboratorios

$$
\bar{x}_{\mathrm{A}}^{2}=\sum \bar{x}_{\mathrm{i}}^{2} / 10
$$

o suma de los valores medios obtenidos por los 10 laboratorios, dividido por 10 que corresponde al número (p) de laboratorios participantes en el ensayo colectivo.

Varianza media del conjunto de laboratorios $\left(s_{\mathrm{A}}^{2}\right)$

$$
s_{\mathrm{A}}^{2}=\Sigma s_{\mathrm{i}}^{2} / 10
$$

o suma de las varianzas obtenidas por los 10 laboratorios, dividido por el número de laboratorios, que recibe también el nombre de varianza media compensada.

Desviación típica media del conjunto de laboratorios $\left(\sigma_{\mathrm{A}}\right)$

$$
\sigma_{\mathrm{A}}=\sqrt{s_{\mathrm{A}}^{2}}
$$

o raiz cuadrada de la varianza media compensada del conjunto de laboratorios.

Coeficiente de variación medio, \% del conjunto de laboratorios $\left(V_{\mathrm{A}}, \%\right)$

$$
V_{\mathrm{A}}(\%)=\left(\sigma_{\mathrm{A}} / \bar{x}_{\mathrm{A}}\right) 100
$$

o cociente entre la desviación típica media y el valor medio global del conjunto de laboratorios, multiplicado por 100 .

\section{Desviación tipica entre laboratorios $\left(\sigma_{\mathrm{EL}}\right)$}

$$
\sigma_{\mathrm{EL}}=\sqrt{s_{\mathrm{A}}^{2}+s_{\mathrm{L}_{\mathrm{A}}}^{2}}
$$

Para el cálculo de la desviación típica entre laboratorios se hace uso del parámetro estadistico $s_{\mathrm{LA}_{\mathrm{A}}}^{2} \mathrm{O}$ 
componente de la varianza entre laboratorios, que se determina a partir de la expresión siguiente:

$$
s_{\mathrm{L}_{\mathrm{A}}}^{2}=s_{\overline{\mathrm{x}}_{\mathrm{A}}}^{2}-\left(s_{\mathrm{A}}^{2} / n\right)
$$

es decir, que $\left(s_{\mathrm{L}_{\mathrm{A}}}^{2}\right)$ es igual a la varianza de las medias de los laboratorios menos la varianza media compensada. En este caso (2), $n=3$.

A su vez, la varianza de las medias de los laboratorios $\left(s_{\overline{\mathrm{x}}_{\mathrm{A}}}^{2}\right)$ se calcula de la expresión siguiente:

$$
s_{\overline{\mathrm{x}}_{\mathrm{A}}}^{2}=\left[\Sigma \bar{x}_{\mathrm{i}}^{2}-p\left(\bar{x}_{\mathrm{A}}\right)^{2}\right] /(p-1)
$$

Coeficiente de variación entre laboratorios $\left(V_{\mathrm{EL}}, \%\right)$

$$
V_{\mathrm{EL}}(\%)=\left(\sigma_{\mathrm{EL}} / \bar{x}_{\mathrm{A}}\right) 100
$$

o cociente entre la desviación típica entre laboratorios y el valor medio global del conjunto de laboratorios, multiplicado por 100 .

\subsection{Cuadros resumen}

A continuación se facilita en el siguiente Cuadro I de valores un resumen de los parámetros estadísticos obtenidos por cálculo de los datos del ensayo colectivo.

Asimismo, en este Cuadro se incluyen las especificaciones relativas a la precisión de la medida, dadas en la norma CEN correspondiente. Estas especificaciones están referidas: a los coeficientes de variación en $\%$, tanto para la repetibilidad de resultados en un laboratorio, como para la reproducibilidad de éstos entre laboratorios, y se determinan a partir de los resultados del ensayo de resistencias mecánicas a compresión a la edad de 28 días cuyo cálculo se efectúa

\begin{tabular}{|c|c|c|c|c|c|c|c|}
\hline & \multirow{3}{*}{$\begin{array}{l}\text { Parámetros } \\
\text { estadisticos } \\
\text { calculados }\end{array}$} & \multirow{3}{*}{$\begin{array}{c}\text { Modalidad } \\
\text { de cálculo } \\
\text { considerada }\end{array}$} & \multicolumn{4}{|c|}{ CEMENTO } & \multirow{3}{*}{$\begin{array}{c}\text { Especificaciones } \\
\text { según norma CEN } \\
\text { (para resultados } \\
\text { a } 28 \text { días) }\end{array}$} \\
\hline & & & \multicolumn{2}{|c|}{ PA-350 } & \multicolumn{2}{|c|}{ P-450 ARI } & \\
\hline & & & 7 dias & 28 dias & 7 dias & 28 dias & \\
\hline \multirow{4}{*}{$\begin{array}{l}\text { Desviacion } \\
\text { tipica }\end{array}$} & \multirow{2}{*}{$\begin{array}{l}\text { En un laboratorio } \\
\text { (repetibilidad) }\end{array}$} & (1) & 9.18 & 11.36 & 13.05 & 13.60 & \\
\hline & & $(2)$ & 4.67 & 7.61 & 8.27 & 7.54 & \\
\hline & \multirow{2}{*}{$\begin{array}{l}\text { Entre laboratorios } \\
\text { (reproducibilidad) }\end{array}$} & (1) & 16.43 & 18.29 & 18.38 & 20.12 & \\
\hline & & (2) & 14.31 & 15.85 & 14.92 & 16.37 & \\
\hline \multirow{4}{*}{$\begin{array}{l}\text { Coeficiente } \\
\text { de } \\
\text { rariacion } \\
\%\end{array}$} & \multirow{2}{*}{$\begin{array}{l}\text { En un laboratorio } \\
\text { (repetibilidad) }\end{array}$} & (1) & 2.82 & 2.74 & 3.11 & 2.70 & \\
\hline & & $(2)$ & 1.43 & 1.83 & 1.97 & 9.80 & 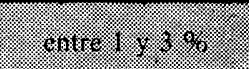 \\
\hline & \multirow{2}{*}{$\begin{array}{l}\text { Entre laboratorios } \\
\text { (reproducibilidad) }\end{array}$} & (1) & 5.04 & 4.41 & 4.39 & 3.99 & \\
\hline & & (2) & 4.39 & 3.82 & 3.56 & 3.25 & I.t. \\
\hline
\end{tabular}
según la modalidad (2).

\section{CUADRO I}

(1) Datos obtenidos a partir de 18 probetas ensayadas a compresion.

12) Datos obtenides a partir de los 3 resultados de las 3 series (de 6 probetas por serie) ensayadas. 
A partir de los anteriores parámetros se ha calculado el Cuadro II de valores, que contiene los intervalos de valores, expresados en $\mathrm{kp} / \mathrm{cm}^{2}$, entre los que previsiblemente pueden oscilar los resultados anteriores, cuando se ensayan:

- en un laboratorio promedio equivalente a los que han intervenido en el ensayo colectivo (repetibilidad), y

- entre los expresados laboratorios (reproducibilidad).

Completan este Cuadro los valores extremos entre los que podrian oscilar los anteriores resultados según los márgenes de dispersión máximos admitidos por la norma CEN.

\section{CUADRO II}

\begin{tabular}{|c|c|c|c|c|c|c|c|}
\hline \multirow{3}{*}{$\begin{array}{l}\text { Cemento } \\
\text { ensayado }\end{array}$} & \multirow{3}{*}{$\begin{array}{c}\text { Resistencias me- } \\
\text { cánicas a com- } \\
\text { presión a } 28 \text { dias } \\
\text { en } \mathbf{k p} / \mathbf{c m}^{2} \\
\overline{\mathbf{x}}_{\mathbf{A}}\end{array}$} & \multicolumn{4}{|c|}{ Coeficiente de variación } & \multirow{2}{*}{\multicolumn{2}{|c|}{$\begin{array}{c}\begin{array}{c}\text { Intervalo } \\
\text { de variación } \\
\text { de la medida }\end{array} \\
\text { Valores }\end{array}$}} \\
\hline & & \multirow{2}{*}{\multicolumn{2}{|c|}{ Parámetros estadisticos calculados }} & \multirow{2}{*}{$\begin{array}{l}\text { en } \\
\%\end{array}$} & \multirow{2}{*}{$\begin{array}{c}\text { en } \\
\mathrm{kp} / \mathrm{cm}^{2}\end{array}$} & & \\
\hline & & & & & & minimo & máximo \\
\hline \multirow{4}{*}{ PA-350 } & \multirow{4}{*}{415} & \multirow{2}{*}{$\begin{array}{l}\text { En un laboratorio } \\
\text { (repetibilidad) }\end{array}$} & Ensayo interlab. & 1,83 & 7,59 & 407 & 422 \\
\hline & & & Valor máx. según CEN & 3 & 12,44 & 402 & 427 \\
\hline & & \multirow{2}{*}{$\begin{array}{l}\text { Entre laboratorios } \\
\text { (reproducibilidad) }\end{array}$} & Ensayo interlab. & 3,82 & 15,84 & 399 & 431 \\
\hline & & & Valor máx. según $\mathrm{CEN}$ & 6 & 24,88 & 390 & 440 \\
\hline \multirow{4}{*}{ P-450 ARI } & \multirow{4}{*}{504} & \multirow{2}{*}{$\begin{array}{l}\text { En un laboratorio } \\
\text { (repetibilidad) }\end{array}$} & Ensayo interlab. & 1.50 & 7,55 & 496 & 511 \\
\hline & & & Valor máx. según CEN & 3 & 15.11 & 489 & 519 \\
\hline & & \multirow{2}{*}{$\begin{array}{l}\text { Entre laboratorios } \\
\text { (reproducibilidad) }\end{array}$} & Ensayo interlab. & 3.25 & 16.37 & 487. & 520 \\
\hline & & & Valor máx. según CEN & 6 & 30,22 & 474 & 534 \\
\hline
\end{tabular}

1)

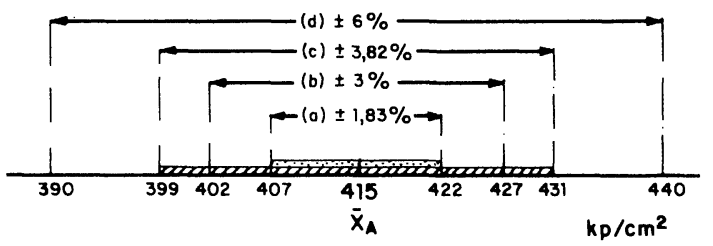

2)

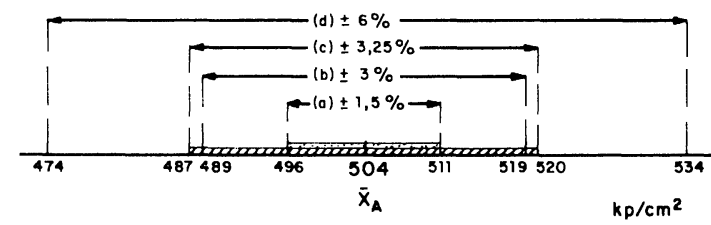

Fig. 1.-Resistencias mecánicas a compresión. Intervalos de variación de la medida en el Ensayo Colectivo.

a) Coef. de variación, \% en un laboratorio (repetibilidad) en el Ensayo Colectivo.

1) Cemento PA-350 a 28 dias.

b) Coef. de variación, \% en un laboratorio, máximo admitido según norma CEN.

c) Coef. de variacion. \% entre laboratorios (reproducibilidad) en el Ensayo Colectivo.

2) Cemento P-450 ARI a 28 dias

d) Coef. de variación. \% entre laboratorios. máximo admitido según norma CEN. 


\section{COMENTARIOS}

Del estudio realizado se deducen los comentarios siguientes:

5.1. Que, como puede observarse en el Cuadro I, las desviaciones tipicas y los coeficientes de variación, hallados según la modalidad de cálculo (1) son, en todos los casos, superiores a los que resultan del cálculo según la modalidad (2).

Esto se explica fácilmente porque en el primer caso los datos han sido obtenidos a partir de 18 valores individuales de resistencia mecánica a compresión, cuya dispersión es muy superior a la que resulta cuando estos mismos valores están agrupados en series de 6 valores (que corresponden al modo de operar en el ensayo normalizado), lo que produce un efecto de compensación en las dispersiones de la serie.

5.2. Que los valores de los coeficientes de variación en tanto por ciento en un laboratorio (promedio), obtenidos en el ensayo colectivo a la edad de 28 días en los cementos estudiados |calculados según (2)| -expresión de la repetibilidad del resultado-, están comprendidos entre: 1.50 y $1,83 \%$.

Por tanto, son muy inferiores al valor máximo del $3 \%$ admitido en la norma CEN en estudio.

5.3. Que lo mismo sucede con los coeficientes de variación en tanto por ciento entre laboratorios obtenidos en el ensayo colectivo -expresión de la reproducibilidad-, cuyos valores están comprendidos entre: 3,25 y $3,82 \%$.

Que resultan también inferiores al valor del $6 \%$ que como máximo admite la norma CEN de referencia.

5.4. Que previsiblemente estos valores, a pesar de que están situados por debajo de los márgenes admitidos, pueden ser mejorados cuando se opere con la instrumentación más precisa propuesta en la norma UNE, anteriormente mencionada, de conformidad con la CEN.

5.5. Que, más concretamente, el intervalo de valores en cuyo entorno previsiblemente pueden oscilar los resultados concretos de las resistencias mecánicas a compresión a la edad de 28 dias (edad considerada en la norma CEN en cuestión), de los dos cementos ensayados en este trabajo, calculado este intervalo de los datos estadisticos anteriores, es:

- para los obtenidos en un laboratorio promedio, tal como los que han intervenido en el ensayo colectivo, los siguientes:

\begin{tabular}{l}
\multicolumn{1}{c}{ Cementos } \\
PA-350 \\
P-450 ARI
\end{tabular}

\section{Intervalo de variación}

de 407 a $422 \mathrm{kp} / \mathrm{cm}^{2}$ de 496 a $511 \mathrm{kp} / \mathrm{cm}^{2}$

- para los obtenidos entre los laboratorios que componen el colectivo de laboratorios que han participado en el ensayo colectivo:

$\frac{\text { Cementos }}{\text { PA-350 }}$
P-450 ARI

Materiales dF. Construccion N. ${ }^{\circ} 192-1983$
Intervalo de variación

de 399 a $431 \mathrm{kp} / \mathrm{cm}^{2}$ de 487 a $520 \mathrm{kp} / \mathrm{cm}^{2}$ 
Valores que se destacan a título indicativo, como exponente de los órdenes de mágnitud en que oscila la precisión de la medida.

5.6. Por último, que los entornos admitidos por las normas CEN y UNE de referencia para la dispersión de los resultados del ensayo, son lo suficientemente amplios para que, en líneas generales, los resultados obtenidos por un laboratorio experimentado, dotado con el instrumental idóneo, queden con facilidad dentro de dichos entornos y alejados apreciablemente de los valores extremos.

\section{BIBLIOGRAFIA}

(1) Pliego de prescripciones técnicas generales para la recepción de cementos RC-75 (B.O.E. n. 206 de 28 de agosto de 1975).

(2) Norme européenne prEN 196 methodes d'essais des ciments. Déterminations des résistances mécaniques

(3) Norma ASTM C 802-80 Conducting an Interlaboratory Test Program to determine the Precision of Test Methods for Constructional Materials.

(4) Norma ASTM C 670-80. Preparing Precision Statements for Test Methods for Construction Materials.

(5) Norma AFNOR X 06-041. Fidélité d'une méthode d'essais. Guide pour les essais interlaboratoires (E). marzo 1970.

(6) Norma ISO 5725. Fidélité des méthodes d'essai. Déterminations de la répétabilité et de la reproductibilité par essai interlaboratoire. 1. ${ }^{\circ}$ Ed. abril 1981.

(7) BAUR, E. L.: Manual de estadística para químicos. Ed. Alhambra (1974).

(8) VENUAT y PAPADAKIS: Control de Ensayos de Cementos. Morteros y Hormigones. Ed. Urmo (1966).

(9) FISHER, R. A.: Métodos estadisticos para investigadores. Ed. Aguilar.

(10) CHAPOUILLE, P. y PAZZIS, R.: Fiabilité des systèmes. Les techniques de base de l'informatique (1968).

\section{A G R A D E C I M I E N T O}

El autor agradece a los Sres. Hidalgo y Aguanell, Presidente y Vocal respectivamente del G.T. 1 de la Comisión Técnica 80 de IRANOR, las observaciones formuladas al texto del artículo. 
CUADRO III

Ensayo interlaboratorio. Resistencias mecánicas a compresión, $\mathrm{Kp} / \mathrm{cm}^{2}$

Cemento: PA-350

Edad: 7 dias

\begin{tabular}{|c|c|c|c|c|c|c|c|c|c|}
\hline $\begin{array}{l}\text { LAB. } \\
\mathrm{N}^{\circ}\end{array}$ & & VALOR & ES INDIVID & UALES & & & $\begin{array}{c}\text { MEDIA } \\
\overline{\mathbf{x}}_{\mathbf{i}}\end{array}$ & $\begin{array}{c}\text { VARIANZA } \\
\mathbf{s}_{\mathbf{i}}^{2}\end{array}$ & DES. MED. \% \\
\hline 1 & $\begin{array}{l}349.00 \\
310.00 \\
353.00\end{array}$ & $\begin{array}{l}345.00 \\
321.00 \\
335.00\end{array}$ & $\begin{array}{l}336.00 \\
340.00 \\
353.00\end{array}$ & $\begin{array}{l}353.00 \\
345.00 \\
345.00\end{array}$ & $\begin{array}{l}355.00 \\
339.00 \\
326.00\end{array}$ & $\begin{array}{l}352.00 \\
345.00 \\
333.00\end{array}$ & 341.17 & 132.74 & 4.61 \\
\hline 2 & $\begin{array}{l}337.00 \\
325.00 \\
319.00\end{array}$ & $\begin{array}{l}319.00 \\
331.00 \\
325.00\end{array}$ & $\begin{array}{l}319.00 \\
331.00 \\
319.00\end{array}$ & $\begin{array}{l}300.00 \\
315.00 \\
328.00\end{array}$ & $\begin{array}{l}32: .00 \\
312.00 \\
312.00\end{array}$ & $\begin{array}{l}331.00 \\
306.00 \\
312.00\end{array}$ & 320.89 & 97.99 & -1.61 \\
\hline 3 & $\begin{array}{l}316.00 \\
3: 5.00 \\
312.00\end{array}$ & $\begin{array}{l}300.00 \\
319.00 \\
325.00\end{array}$ & $\begin{array}{l}321.00 \\
325.00 \\
312.00\end{array}$ & $\begin{array}{l}312.00 \\
325.00 \\
325.00\end{array}$ & $\begin{array}{l}319.00 \\
325.00 \\
319.00\end{array}$ & $\begin{array}{l}319.00 \\
319.00 \\
319.00\end{array}$ & 319.72 & 43.27 & -2.27 \\
\hline 4 & $\begin{array}{l}331.00 \\
332.00 \\
324.00\end{array}$ & $\begin{array}{l}324.00 \\
336.00 \\
335.00\end{array}$ & $\begin{array}{l}336.00 \\
332.00 \\
324.00\end{array}$ & $\begin{array}{l}326.00 \\
338.00 \\
339.00\end{array}$ & $\begin{array}{l}334.00 \\
329.00 \\
328.00\end{array}$ & $\begin{array}{l}322.00 \\
331.00 \\
328.00\end{array}$ & 330.50 & 27.09 & 1.34 \\
\hline 5 & $\begin{array}{l}339.00 \\
332.00 \\
341.00\end{array}$ & $\begin{array}{l}329.00 \\
343.01 \\
340.06\end{array}$ & $\begin{array}{l}339.00 \\
336.00 \\
337.00\end{array}$ & $\begin{array}{l}339.00 \\
339.00 \\
340.00\end{array}$ & $\begin{array}{l}336.00 \\
339.00 \\
330.00\end{array}$ & $\begin{array}{l}341.00 \\
316.00 \\
332.00\end{array}$ & 336.00 & 40.82 & 3.02 \\
\hline 6 & $\begin{array}{l}346.00 \\
338.00 \\
358.00\end{array}$ & $\begin{array}{l}342.00 \\
334.00 \\
350.00\end{array}$ & $\begin{array}{l}346.00 \\
346.00 \\
334.00\end{array}$ & $\begin{array}{l}338.00 \\
342.00 \\
330.00\end{array}$ & $\begin{array}{l}326.00 \\
326.00 \\
342.00\end{array}$ & $\begin{array}{l}346.00 \\
334.00 \\
342.00\end{array}$ & 340.00 & 70.12 & 4.25 \\
\hline 7 & $\begin{array}{l}341.00 \\
331.00 \\
334.00\end{array}$ & $\begin{array}{l}341.00 \\
331.00 \\
337.00\end{array}$ & $\begin{array}{l}341.00 \\
334.00 \\
328.00\end{array}$ & $\begin{array}{l}334.00 \\
328.00 \\
328.00\end{array}$ & $\begin{array}{l}331.00 \\
337.00 \\
341.00\end{array}$ & $\begin{array}{l}337.00 \\
344.00 \\
331.00\end{array}$ & 334.94 & 26.53 & 2.70 \\
\hline 8 & $\begin{array}{l}250.00 \\
290.00 \\
296.00\end{array}$ & $\begin{array}{l}296.00 \\
277.00 \\
308.00\end{array}$ & $\begin{array}{l}290.00 \\
296.00 \\
290.00\end{array}$ & $\begin{array}{l}302.00 \\
253.00 \\
296.00\end{array}$ & $\begin{array}{l}308.00 \\
250.00 \\
308.00\end{array}$ & $\begin{array}{l}290.00 \\
250.00 \\
302.00\end{array}$ & 294.89 & 72.22 & -9.58 \\
\hline 9 & $\begin{array}{l}338.00 \\
328.00 \\
329.00\end{array}$ & $\begin{array}{l}333.00 \\
325.00 \\
325.00\end{array}$ & $\begin{array}{l}324.00 \\
339.00 \\
291.00\end{array}$ & $\begin{array}{l}324.00 \\
329.00 \\
318.00\end{array}$ & $\begin{array}{l}304.00 \\
316.00 \\
339.00\end{array}$ & $\begin{array}{l}335.00 \\
313.00 \\
323.00\end{array}$ & 324.39 & 155.78 & -.54 \\
\hline io & $\begin{array}{l}338.00 \\
325.00 \\
320.00\end{array}$ & $\begin{array}{l}338.00 \\
301.00 \\
306.00\end{array}$ & $\begin{array}{l}336.00 \\
297.00 \\
326.00\end{array}$ & $\begin{array}{l}336.00 \\
300.00 \\
325.00\end{array}$ & $\begin{array}{l}305.00 \\
327.00 \\
323.00\end{array}$ & $\begin{array}{l}323.00 \\
312.00 \\
318.00\end{array}$ & 319.39 & 176.69 & -1.92 \\
\hline
\end{tabular}

$$
\begin{aligned}
& \bar{x}_{\mathrm{A}}=326,14 \\
& s_{\mathrm{A}}^{2}=84,32
\end{aligned}
$$

\begin{tabular}{llll}
\hline DESV. TIP. EN UN LAB. $\left(\sigma_{A}\right)$ & $=9,13$ & (Repctib.) \\
DESV. TIP. ENTRE LABS $\left(\sigma_{E L}\right)$ & $=16,43$ & (Reproducib.) \\
COEF. VAR. EN UN LAB., $\%\left(V_{A} \%\right)=2,82$ & (Repetib.) \\
COEF. VAR. ENTRE LABS., $\%\left(V_{E L} \%\right)=5,04$ & (Reproducib.)
\end{tabular}


CUADRO IV

Ensayo interlaboratorio. Resistencias mecánicas a compresión, $\mathrm{Kp} / \mathrm{cm}^{2}$

Cemento: PA-350

Edad: 28 días

\begin{tabular}{|c|c|c|c|c|c|c|c|c|c|}
\hline $\begin{array}{l}\text { LAB. } \\
\mathrm{N}^{\circ}\end{array}$ & \multicolumn{6}{|c|}{ VALORES INDIVIDUALES } & \multirow{2}{*}{$\begin{array}{l}\frac{\overline{\mathbf{X}}_{\mathbf{i}}}{4 \mathrm{EDIA}} \\
401.50\end{array}$} & \multirow{2}{*}{$\begin{array}{c}\begin{array}{c}\text { VARIANZA } \\
\mathbf{s}_{i}^{2}\end{array} \\
302.15\end{array}$} & \multirow{2}{*}{\begin{tabular}{|c|} 
DES. MED. $\%$ \\
-3.20
\end{tabular}} \\
\hline 1 & $\begin{array}{l}404.00 \\
358.00 \\
406.00\end{array}$ & $\begin{array}{l}415.00 \\
407.00 \\
394.00\end{array}$ & $\begin{array}{l}404.00 \\
36: .00 \\
411.00\end{array}$ & $\begin{array}{l}396.00 \\
388.00 \\
405.00\end{array}$ & $\begin{array}{l}405.00 \\
371.00 \\
409.00\end{array}$ & $\begin{array}{l}439.010 \\
406.00 \\
416.00\end{array}$ & & & \\
\hline 2 & $\begin{array}{l}40 E .00 \\
4 U 6.00 \\
41 Z .010\end{array}$ & $\begin{array}{l}412.00 \\
409.00 \\
375.00\end{array}$ & $\begin{array}{l}412.00 \\
375.00 \\
419.00\end{array}$ & $\begin{array}{l}394.00 \\
356.00 \\
403.00\end{array}$ & $\begin{array}{l}419.00 \\
38 ? .00 \\
41.2 .00\end{array}$ & $\begin{array}{l}412.00 \\
375.00 \\
400.00\end{array}$ & 399.11 & 329.75 & -3.77 \\
\hline 3 & $\begin{array}{l}396.00 \\
413.00 \\
409.00\end{array}$ & $\begin{array}{l}400.00 \\
406.00 \\
413.00\end{array}$ & $\begin{array}{l}408.00 \\
413.00 \\
406.00\end{array}$ & $\begin{array}{l}410.00 \\
413.00 \\
419.00\end{array}$ & $\begin{array}{l}413.00 \\
413.00 \\
410.00\end{array}$ & $\begin{array}{l}415.00 \\
409.00 \\
419.00\end{array}$ & 410.17 & 34.50 & -1.11 \\
\hline 4 & $\begin{array}{l}419.00 \\
425.00 \\
430.00\end{array}$ & $\begin{array}{l}418.00 \\
426.00 \\
418.00\end{array}$ & $\begin{array}{l}420.00 \\
419.00 \\
428.00\end{array}$ & $\begin{array}{l}422.00 \\
423.00 \\
418.00\end{array}$ & $\begin{array}{l}420.00 \\
421.00 \\
422.00\end{array}$ & $\begin{array}{l}415.00 \\
426.00 \\
419.00\end{array}$ & 421.89 & 18.22 & 1.72 \\
\hline 5 & $\begin{array}{r}415.00 \\
433.00 \\
+32.00\end{array}$ & $\begin{array}{l}\$ 21.00 \\
+50.00 \\
435.00\end{array}$ & $\begin{array}{l}422.00 \\
445.00 \\
426.00\end{array}$ & $\begin{array}{l}430.00 \\
237.00 \\
419.00\end{array}$ & $\begin{array}{l}419.00 \\
427.00 \\
430.00\end{array}$ & $\begin{array}{l}429.00 \\
425.00 \\
416.00\end{array}$ & 427.50 & 117.91 & 3.07 \\
\hline 6 & $\begin{array}{r}406.00 \\
413.00 \\
425.00\end{array}$ & $\begin{array}{l}406.00 \\
413.00 \\
421.00\end{array}$ & $\begin{array}{l}410.00 \\
425.00 \\
413.00\end{array}$ & $\begin{array}{l}421.00 \\
429.00 \\
429.00\end{array}$ & $\begin{array}{l}417.00 \\
417.00 \\
429.00\end{array}$ & $\begin{array}{l}406.00 \\
417.00 \\
423.00\end{array}$ & 418.11 & 69.03 & .01 \\
\hline 7 & $\begin{array}{l}431.00 \\
412.00 \\
+12.00\end{array}$ & $\begin{array}{r}428.00 \\
+19.00 \\
+12.00\end{array}$ & $\begin{array}{l}434.00 \\
434.00 \\
425.00\end{array}$ & $\begin{array}{l}425.00 \\
428.00 \\
419.00\end{array}$ & $\begin{array}{l}425.00 \\
434.00 \\
419.00\end{array}$ & $\begin{array}{l}428.00 \\
437.00 \\
422.00\end{array}$ & 424.67 & 63.06 & 2.39 \\
\hline 8 & $\begin{array}{l}377.00 \\
390.60 \\
383.00\end{array}$ & $\begin{array}{l}390.00 \\
390.00 \\
377.00\end{array}$ & $\begin{array}{l}408.00 \\
408.00 \\
390.00\end{array}$ & $\begin{array}{l}396.00 \\
390.00 \\
383.00\end{array}$ & $\begin{array}{l}390.00 \\
396.00 \\
396.00\end{array}$ & $\begin{array}{l}402.00 \\
402.00 \\
377.00\end{array}$ & 331.72 & 95.98 & -5.55 \\
\hline y & $\begin{array}{l}416.00 \\
+13.01 \\
403.00\end{array}$ & $\begin{array}{l}413.00 \\
403.00 \\
419.00\end{array}$ & $\begin{array}{l}420.00 \\
409.00 \\
403.00\end{array}$ & $\begin{array}{l}434.00 \\
425.00 \\
409.00\end{array}$ & $\begin{array}{l}404.00 \\
416.00 \\
415.00\end{array}$ & $\begin{array}{l}398.00 \\
415.00 \\
4: 9.00\end{array}$ & 413.28 & 76.09 & -.36 \\
\hline 10 & $\begin{array}{l}440.00 \\
428.00 \\
436.00\end{array}$ & $\begin{array}{r}438.00 \\
428.00 \\
+30.00\end{array}$ & $\begin{array}{l}449.00 \\
453.00 \\
422.00\end{array}$ & $\begin{array}{l}421.00 \\
453.00 \\
438.00\end{array}$ & $\begin{array}{l}453.00 \\
460.00 \\
425.00\end{array}$ & $\begin{array}{l}458.00 \\
456.00 \\
425.00\end{array}$ & 439.61 & 183.08 & 5.99 \\
\hline
\end{tabular}

$$
\begin{aligned}
& \bar{x}_{\mathrm{A}}=414,76 \\
& s_{\mathrm{A}}^{2}=128,98
\end{aligned}
$$

\begin{tabular}{|lll|}
\hline DESV. TIP. EN UN LAB. $\left(\sigma_{A}\right)$ & $=11,36$ & (Repetib.) \\
DESV. TIP. ENTRE LABS $\left(\sigma_{E L}\right)$ & $=18,29$ & (Reproducib.) \\
COEF. VAR. EN UN LAB., \% $\left(V_{A} \%\right)$ & $=2,74$ & (Repetib.) \\
COEF. VAR. ENTRE LABS. \% $\left(V_{E L} \%\right)$ & $=4,41$ & (Reproducib.)
\end{tabular}




\section{CUADRO V}

Ensayo interlaboratorio. Resistencias mecánicas a compresión, $\mathrm{Kp} / \mathrm{cm}^{2}$

Cemento: P-450 ARI

Edad: $\quad 7$ dias

\begin{tabular}{|c|c|c|c|c|c|c|c|c|c|}
\hline $\begin{array}{c}\text { LAB. } \\
\text { N. }\end{array}$ & \multicolumn{6}{|c|}{ VALORES INDIVIDUALES } & \multirow{2}{*}{$\begin{array}{c}\begin{array}{c}\text { MEDIA } \\
\bar{x}_{i}\end{array} \\
434.50\end{array}$} & \multirow{2}{*}{$\begin{array}{c}\begin{array}{c}\text { VARIANZA } \\
s_{i}^{2}\end{array} \\
241.51\end{array}$} & \multirow{2}{*}{$\begin{array}{r}\text { DES. MED. } \% \\
3.69\end{array}$} \\
\hline 1 & $\begin{array}{l}451.00 \\
454.00 \\
435.00\end{array}$ & $\begin{array}{l}456.00 \\
455.00 \\
434.00\end{array}$ & $\begin{array}{l}414.00 \\
446.00 \\
401.00\end{array}$ & $\begin{array}{l}433.00 \\
433.00 \\
434.00\end{array}$ & $\begin{array}{l}424.00 \\
423.00 \\
433.00\end{array}$ & $\begin{array}{l}416.00 \\
430.00 \\
445.00\end{array}$ & & & \\
\hline 2 & $\begin{array}{l}444.00 \\
406.00 \\
417.00\end{array}$ & $\begin{array}{l}457=00 \\
375.00 \\
406.00\end{array}$ & $\begin{array}{l}444.00 \\
327.00 \\
428.00\end{array}$ & $\begin{array}{l}416.00 \\
387.00 \\
466.00\end{array}$ & $\begin{array}{l}415.00 \\
425.00 \\
456.00\end{array}$ & $\begin{array}{l}410.00 \\
412.00 \\
419.00\end{array}$ & 414.00 & 756.94 & -1.20 \\
\hline 3 & $\begin{array}{l}425.00 \\
425.00 \\
421.00\end{array}$ & $\begin{array}{l}4: 2.00 \\
425.00 \\
429.00\end{array}$ & $\begin{array}{l}432.00 \\
431.00 \\
432.00\end{array}$ & $\begin{array}{l}432.00 \\
429.00 \\
432.00\end{array}$ & $\begin{array}{l}419.00 \\
419.00 \\
419.00\end{array}$ & $\begin{array}{l}412.00 \\
412.00 \\
419.00\end{array}$ & 423.61 & 52.72 & 1.03 \\
\hline 4 & $\begin{array}{l}420.00 \\
422.00 \\
415.00\end{array}$ & $\begin{array}{l}415.00 \\
421.00 \\
426.00\end{array}$ & $\begin{array}{l}419.00 \\
415.00 \\
428.00\end{array}$ & $\begin{array}{l}420.00 \\
411.00 \\
414.00\end{array}$ & $\begin{array}{l}412.00 \\
410.00 \\
411.03\end{array}$ & $\begin{array}{l}415.00 \\
419.00 \\
408.00\end{array}$ & 418.22 & 27.95 & -.43 \\
\hline$s$ & $\begin{array}{l}435.00 \\
419.00 \\
440.00\end{array}$ & $\begin{array}{l}444.00 \\
221.00 \\
442.00\end{array}$ & $\begin{array}{l}437.00 \\
439.00 \\
428.00\end{array}$ & $\begin{array}{l}429.00 \\
438.00 \\
451.00\end{array}$ & $\begin{array}{l}422.00 \\
453.00 \\
414.00\end{array}$ & $\begin{array}{l}416.05 \\
437.00 \\
42.00\end{array}$ & 432.72 & 135.51 & 3.27 \\
\hline 6 & $\begin{array}{l}421.00 \\
401.00 \\
409.00\end{array}$ & $\begin{array}{l}385.00 \\
41 ? .00 \\
421.00\end{array}$ & $\begin{array}{l}429.00 \\
429.00 \\
417.00\end{array}$ & $\begin{array}{l}417.00 \\
410.00 \\
414.00\end{array}$ & $\begin{array}{l}414.00 \\
399.00 \\
414.00\end{array}$ & $\begin{array}{l}410.00 \\
398.00 \\
421.00\end{array}$ & 412.56 & 124.03 & -1.53 \\
\hline 7 & $\begin{array}{l}447.00 \\
441.00 \\
437.00\end{array}$ & $\begin{array}{l}441.00 \\
437.00 \\
434.00\end{array}$ & $\begin{array}{l}444.00 \\
44 i .00 \\
431.00\end{array}$ & $\begin{array}{l}437.00 \\
444.00 \\
431.00\end{array}$ & $\begin{array}{l}441.00 \\
437.00 \\
425.00\end{array}$ & $\begin{array}{l}437.00 \\
141.00 \\
425.00\end{array}$ & 437.28 & 37.98 & 4.35 \\
\hline 8 & $\begin{array}{l}396.00 \\
296.00 \\
402.00\end{array}$ & $\begin{array}{l}41500 \\
383.00 \\
390.00\end{array}$ & $\begin{array}{l}402.00 \\
333.00 \\
383.00\end{array}$ & $\begin{array}{l}402.00 \\
377.00 \\
383.00\end{array}$ & $\begin{array}{l}396.00 \\
396.00 \\
396.00\end{array}$ & $\begin{array}{l}383.00 \\
402.00 \\
409.00\end{array}$ & 394.06 & 107.23 & -5.36 \\
\hline 9 & $\begin{array}{l}421.00 \\
415.00 \\
406.00\end{array}$ & $\begin{array}{l}421.00 \\
406.00 \\
400.00\end{array}$ & $\begin{array}{l}428.00 \\
433.00 \\
418.00\end{array}$ & $\begin{array}{l}421.00 \\
428.00 \\
418.00\end{array}$ & $\begin{array}{l}413.00 \\
425.00 \\
406.00\end{array}$ & $\begin{array}{l}394.00 \\
418.00 \\
400.00\end{array}$ & 415.67 & 132.00 & -.80 \\
\hline 10 & $\begin{array}{l}295.00 \\
413.00 \\
425.00\end{array}$ & $\begin{array}{l}408.00 \\
414.00 \\
418.00\end{array}$ & $\begin{array}{l}400.00 \\
412.00 \\
413.00\end{array}$ & $\begin{array}{l}401.00 \\
406.00 \\
419.00\end{array}$ & $\begin{array}{l}394.00 \\
401.00 \\
414.00\end{array}$ & $\begin{array}{l}396.00 \\
407.00 \\
416.00\end{array}$ & 408.72 & 85.51 & -2.46 \\
\hline
\end{tabular}

$$
\begin{aligned}
& \bar{x}_{\mathrm{A}}=419,03 \\
& s_{\mathrm{A}}^{2}=170,18
\end{aligned}
$$

$\begin{array}{lll}\text { DESV. TIP. EN UN LAB. }\left(\sigma_{A}\right) & =13,05 & \text { (Repetib.) } \\ \text { DESV. TIP. ENTRE LABS }\left(\sigma_{E L}\right) & =18,38 & \text { (Reproducib.) } \\ \text { COEF. VAR. EN UN LAB., } \%\left(V_{A} \%\right)=3,11 & \text { (Repetib.) } \\ \text { COEF. VAR. ENTRE LABS., } \%\left(V_{E L} \%\right)=4,39 & \text { (Reproducib.) }\end{array}$

Matfriales dF. CONStruccion N. ${ }^{\circ} 192-1983$ 
CUADRO VI

Ensayo interlaboratorio. Resistencias mecánicas a compresión, $\mathrm{Kp} / \mathrm{cm}^{2}$

Cemento: P-450 ARI

Edad: 28 dias

\begin{tabular}{|c|c|c|c|c|c|c|c|c|c|}
\hline $\begin{array}{c}\text { LAB. } \\
\mathrm{N}^{\circ}\end{array}$ & \multicolumn{6}{|c|}{ VALORES INDIVIDUALES } & $\begin{array}{c}\text { MEDIA } \\
\overline{\mathbf{x}}_{\mathbf{i}}\end{array}$ & $\begin{array}{c}\text { VARIANZA } \\
\mathbf{s}_{\mathbf{i}}^{2}\end{array}$ & DES. MED. $\%$ \\
\hline i & $\begin{array}{l}480.00 \\
580.00 \\
408.00\end{array}$ & $\begin{array}{l}509.00 \\
483.00 \\
455.00\end{array}$ & $\begin{array}{l}509.00 \\
490.00 \\
473.00\end{array}$ & $\begin{array}{l}471.00 \\
504.00 \\
490.00\end{array}$ & $\begin{array}{l}460.00 \\
477.00 \\
469.00\end{array}$ & $\begin{array}{l}462.00 \\
493.0= \\
476.00\end{array}$ & 434.50 & 239.09 & -3.82 \\
\hline 2 & $\begin{array}{l}519.00 \\
482.00 \\
487.010\end{array}$ & $\begin{array}{l}506.00 \\
469.00 \\
491.00\end{array}$ & $\begin{array}{l}487.00 \\
475.00 \\
537.00\end{array}$ & $\begin{array}{l}491.00 \\
494.00 \\
505.00\end{array}$ & $\begin{array}{l}422.00 \\
475.00 \\
537.00\end{array}$ & $\begin{array}{l}500.00 \\
487.00 \\
512.00\end{array}$ & 491.50 & 749.32 & -2.43 \\
\hline 3 & $\begin{array}{l}505.00 \\
509.00 \\
513.00\end{array}$ & $\begin{array}{l}506.00 \\
507.00 \\
509.00\end{array}$ & $\begin{array}{l}515.00 \\
503.00 \\
504.00\end{array}$ & $\begin{array}{l}510.00 \\
498.00 \\
515.00\end{array}$ & $\begin{array}{l}503.00 \\
503.00 \\
519.00\end{array}$ & $\begin{array}{l}511.00 \\
505.00 \\
513.00\end{array}$ & 508.72 & 26.45 & $: 93$ \\
\hline 4 & $\begin{array}{l}456.00 \\
452.00 \\
495.00\end{array}$ & $\begin{array}{l}509.00 \\
459.00 \\
438.00\end{array}$ & $\begin{array}{l}494.00 \\
505.00 \\
490.00\end{array}$ & $\begin{array}{l}436.00 \\
508.00 \\
481.00\end{array}$ & $\begin{array}{l}488.00 \\
455.00 \\
488.00\end{array}$ & $\begin{array}{l}490.00 \\
478.00 \\
480.00\end{array}$ & 495.78 & 48.30 & $-i . \equiv Q$ \\
\hline 5 & $\begin{array}{l}525.00 \\
545.00 \\
525.00\end{array}$ & $\begin{array}{l}547.00 \\
556.00 \\
506.00\end{array}$ & $\begin{array}{l}530.00 \\
510.00 \\
535.00\end{array}$ & $\begin{array}{l}5<? .00 \\
555.00 \\
520.00\end{array}$ & $\begin{array}{l}517.00 \\
516.00 \\
520.00\end{array}$ & $\begin{array}{l}512.00 \\
520.00 \\
5 \geq 0.00\end{array}$ & 329.22 & 245.01 & \$. 05 \\
\hline 6 & $\begin{array}{l}501.00 \\
4 \div 3.00 \\
461.00\end{array}$ & $\begin{array}{l}501.00 \\
493.00 \\
481.00\end{array}$ & $\begin{array}{l}503.00 \\
501.00 \\
497.00\end{array}$ & $\begin{array}{l}510.00 \\
505.00 \\
508.00\end{array}$ & $\begin{array}{l}493.00 \\
425.00 \\
485.00\end{array}$ & $\begin{array}{l}504.00 \\
499.00 \\
493.00\end{array}$ & 494.83 & 145.28 & -1.78 \\
\hline 7 & $\begin{array}{l}512.00 \\
515.00 \\
512.00\end{array}$ & $\begin{array}{l}519.00 \\
525.00 \\
519.00\end{array}$ & $\begin{array}{l}525.00 \\
525.00 \\
509.00\end{array}$ & $\begin{array}{l}528.00 \\
528.00 \\
512.00\end{array}$ & $\begin{array}{l}528.00 \\
528.00 \\
515.00\end{array}$ & $\begin{array}{l}528.00 \\
531.00 \\
515.00\end{array}$ & 521.22 & 48.89 & 3.47 \\
\hline 8 & $\begin{array}{l}508.00 \\
477.00 \\
477.00\end{array}$ & $\begin{array}{l}496.00 \\
477.00 \\
455.00\end{array}$ & $\begin{array}{l}496.40 \\
496.00 \\
490.00\end{array}$ & $\begin{array}{l}483.00 \\
483.00 \\
483.00\end{array}$ & $\begin{array}{l}490.00 \\
490.00 \\
433.00\end{array}$ & $\begin{array}{l}496.00 \\
496.00 \\
483.00\end{array}$ & 487.17 & 102.15 & -3.30 \\
\hline 9 & $\begin{array}{l}504.00 \\
456.00 \\
504.00\end{array}$ & $\begin{array}{l}508.00 \\
504.00 \\
500.00\end{array}$ & $\begin{array}{l}514.00 \\
519.00 \\
501.00\end{array}$ & $\begin{array}{l}516.00 \\
525.00 \\
503.00\end{array}$ & $\begin{array}{l}521.00 \\
521.00 \\
514.00\end{array}$ & $\begin{array}{l}522.00 \\
490.00 \\
513.00\end{array}$ & $=10.39$ & 85.43 & 1.31 \\
\hline 10 & $\begin{array}{l}526.00 \\
525.00 \\
523.00\end{array}$ & $\begin{array}{l}538.00 \\
513.00 \\
512.02\end{array}$ & $\begin{array}{l}505.00 \\
517.00 \\
505.00\end{array}$ & $\begin{array}{l}520.00 \\
530.00 \\
506.00\end{array}$ & $\begin{array}{l}494.00 \\
499.00 \\
522.00\end{array}$ & $\begin{array}{l}511.00 \\
491.00 \\
515.00\end{array}$ & $5: 4.28$ & 158.57 & $2 \cdot 33$ \\
\hline
\end{tabular}

$$
\begin{aligned}
& \bar{x}_{\mathrm{A}}=503,77 \\
& s_{\mathrm{A}}^{2}=184,85
\end{aligned}
$$

\begin{tabular}{|c|c|c|}
\hline DESV. TIP. EN UN LAB. $\left(\sigma_{A}\right)$ & $=13,60$ & (Repetib.) \\
\hline DESV. TIP. ENTRE LABS $\left(\sigma_{E L}\right)$ & $=20,12$ & (Reproduclb.) \\
\hline COEF. VAR. EN UN LAB., \% $\left(V_{A} \%\right)$ & $=2,70$ & (Rapelib.) \\
\hline COEF VAR. ENTRE LABS., $\%\left(V_{E L} \%\right)$ & $=3,99$ & (Reproducib.) \\
\hline
\end{tabular}


CUADRO VII

Ensayo interlaboratorio. Resistencias mecánicas a compresión, $\mathrm{Kp} / \mathrm{cm}^{2}$

Cemento: PA-350

Edad: $\quad 7$ días

\begin{tabular}{|c|c|c|c|c|c|c|}
\hline LAB. $\mathbf{N}^{\circ}$ & \multicolumn{3}{|c|}{ RESULTADOS } & $\overline{\mathbf{x}}_{\mathbf{i}}$ & $\begin{array}{c}\text { VARIANZA } \\
\mathbf{s}^{2}\end{array}$ & DES. MED. $\%$ \\
\hline 1 & 345.32 & 334.53 & 340.55 & 341.10 & 47.08 & 4.61 \\
\hline 2 & 327.83 & 320.67 & 319.17 & 320.89 & 3.39 & -1.61 \\
\hline 3 & $3: 2.50$ & 323.00 & $3: 8.57$ & 318.72 & 19.06 & -2.27 \\
\hline 0 & 328.83 & 333.00 & 329.67 & 330.50 & 4.86 & 2.36 \\
\hline 5 & $3 \pm 7.17$ & 334.17 & 330.67 & 336.00 & 2.58 & 3.02 \\
\hline 6 & 340.67 & 336.67 & 342.67 & 340.03 & 9.33 & 4.25 \\
\hline 7 & 337.50 & 334.17 & 333.17 & 334.95 & 5.10 & 2.70 \\
\hline 8 & 290.00 & 288.67 & 300.00 & 294.89 & 3.3 .02 & $-9.5 i$ \\
\hline 9 & 320.33 & 325.00 & 321.83 & 3211.39 & 5.34 & -.54 \\
\hline 13 & 329.33 & 310.07 & 319.07 & 319.80 & 87.09 & $0 ! .92$ \\
\hline
\end{tabular}

$\bar{x}_{\mathrm{A}}=326,14$

$s_{\mathrm{A}}^{2}=21,79$

$\begin{array}{llll}\text { DESV. TIP. EN UN LAB. }\left(\sigma_{A}\right) & =4,67 & \text { (Repetib.) } \\ \text { DESV. TIP. ENTRE LABS }\left(\sigma_{E L}\right) & =14,31 & \text { (Reproducib.) } \\ \text { COEF. VAR. EN UN LAB., \% }\left(V_{A} \%\right) & =1,43 & \text { (Repetib.) } \\ \text { COEF VAR. ENTRE LABS.,\% }\left(V_{E L} \%\right)=4,39 & \text { (Reproduclb.) }\end{array}$

Mattikiales de: Construccion N. ${ }^{\circ} 192-1983$ 
CUADRO VIII

Ensayo interlaboratorio. Resistencias mecánicas a compresión, $\mathrm{Kp} / \mathrm{cm}^{2}$

Cemento: PA-350

Edad: 28 días

\begin{tabular}{|c|c|c|c|c|c|c|}
\hline LAB. N. ${ }^{\circ}$ & \multicolumn{3}{|c|}{ RESULTADOS } & $\begin{array}{l}\text { MEDIA } \\
\overline{\mathbf{x}}_{\mathbf{i}}\end{array}$ & $\begin{array}{c}\text { VARIANZA } \\
\mathbf{s}_{i}^{2}\end{array}$ & DES. MED. $\%$ \\
\hline 1 & 410.50 & $3 \times 6.83$ & 407.17 & $40: .50$ & 164.18 & -3.20 \\
\hline 2 & 400.17 & 384.67 & 403.50 & 399.11 & 164.49 & -3.77 \\
\hline 3 & 400.67 & 411.17 & 412.67 & 410.17 & 9.75 & -1.91 \\
\hline$\Delta$ & 419.00 & 424.17 & 422.50 & 421.89 & 6.96 & 1.72 \\
\hline 5 & 422.67 & 433.50 & 420.33 & 427.50 & 30.35 & 3.07 \\
\hline 0 & $4 ! 1.00$ & 490.00 & 424.33 & 418.11 & 45.02 & .91 \\
\hline 7 & 428.50 & 427.33 & 419.17 & 424.67 & 32.00 & 2.39 \\
\hline 8 & 393.73 & 397.00 & 384.33 & 391.72 & 43.47 & -5.55 \\
\hline 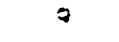 & 414.17 & 413.50 & 412.17 & 413.28 & 1.04 &.- .36 \\
\hline & 443.17 & 440.33 & 429.33 & $43 \div .61$ & 81.76 & 5.99 \\
\hline
\end{tabular}

$\bar{x}_{\mathrm{A}}=414,76$

$s_{\mathrm{A}}^{2}=57,90$

$\begin{array}{lcll}\text { DESV. TIP. EN UN LAB. }\left(\sigma_{A}\right) & =7,61 & \text { (Repetib.) } \\ \text { DESV. TIP. ENTRE LABS }\left(\sigma_{E !}\right) & =15,85 & \text { (Reproducib.) } \\ \text { COEF. VAR. EN UN LAB., } \%\left(V_{A} \%\right)=1,83 & \text { (Repetib.) } \\ \text { COEF. VAR. ENTRE. LABS.\% } \%\left(V_{E L} \%\right)=3,82 & \text { (Reproducib.) }\end{array}$


CUADRO IX

Ensayo interlaboratorio. Resistencias mecánicas a compresión, $\mathrm{Kp} / \mathrm{cm}^{2}$

Cemento: P-450 ARI

Edad: 7 dias

\begin{tabular}{|c|c|c|c|c|c|c|}
\hline LAB. N." & \multicolumn{3}{|c|}{ RESULTADOS } & $\frac{\text { MEDIA }}{\bar{x}_{i}}$ & $\begin{array}{c}\text { VARIANZA } \\
\mathbf{s}_{i}^{2}\end{array}$ & DES. MED. $\%$ \\
\hline 1 & $4 \overline{3} \cdot 33$ & 440.83 & 430.33 & 434.50 & $3 ! .08$ & 3.67 \\
\hline 2 & 429.33 & 300.33 & 422.33 & 414.00 & 432.31 & -1.20 \\
\hline 3 & 422.00 & 423.50 & 425.33 & $423.6 !$ & 2.78 & 1.09 \\
\hline 4 & 416.83 & 417.83 & 417.00 & 417.22 & .28 & .0 .43 \\
\hline 5 & 430.50 & 434.50 & 433.17 & 432.72 & 9.15 & 3.27 \\
\hline 6 & 412.83 & 404.33 & 416.00 & 412.55 & 12.91 & -1.55 \\
\hline 7 & 441.17 & 440.17 & 430.50 & 437.28 & 34.73 & 4.35 \\
\hline 8 & 395.00 & 380.50 & 303.67 & 304.06 & 22.67 & -5.80 \\
\hline 9 & 417.33 & 421.67 & 40.00 & 415.67 & $4 \therefore .79$ & $-.8 v$ \\
\hline 10 & 399.00 & $40 F .83$ & 418.33 & $408.7 ?$ & 93.42 & $=2.40$ \\
\hline
\end{tabular}

$\bar{x}_{\mathrm{A}}=419,03$

$s_{\mathrm{A}}^{2}=68,32$

$\begin{array}{lll}\text { DESV. TIP. EN UN LAB. }\left(\sigma_{A}\right) & =8,27 & \text { (Repelib.) } \\ \text { DESV. TIP. ENTRE LABS }\left(\sigma_{E L}\right) & =14,92 & \text { (Reproducib.) } \\ \text { COEF. VAR. EN UN LAB., \% }\left(V_{A} \%\right)=1,97 & \text { (Repelib.) } \\ \text { COEF. VAR. ENTRE LABS., } \%\left(V_{E L} \%\right)=3,56 & \text { (Reproducib.) }\end{array}$

MATERIAles de Construccion N. ${ }^{\circ} 192-1983$

29 
CUADRO X

Ensayo interlaboratorio. Resistencias mecánicas a compresión, $\mathrm{Kp} / \mathrm{cm}^{2}$

Cemento: P-450 ARI

Edad: 28 dias

\begin{tabular}{|c|c|c|c|c|c|c|}
\hline LAB. N. ${ }^{\circ}$ & \multicolumn{3}{|c|}{ RESULTADOS } & $\begin{array}{l}\text { MEDIA } \\
\overline{\mathbf{x}}_{\mathbf{i}}\end{array}$ & $\begin{array}{c}\text { VARIANZA } \\
\mathbf{s}^{2}\end{array}$ & DES. MED. $\%$ \\
\hline 1 & 482.83 & 493.17 & 477.50 & 484.50 & 63.43 & -3.82 \\
\hline 2 & 487.50 & 477.00 & 510.00 & $49 ! .50$ & $\ddot{204.25}$ & -2.43 \\
\hline 3 & 509.3 .3 & 504.67 & $512.1 ?$ & 508.76 & 14.34 & .98 \\
\hline 4 & 476.83 & 498.83 & 471.07 & 495.78 & 13.65 & -1.57 \\
\hline 5 & 550.33 & 533.233 & 523.50 & 529.22 & 27.60 & 5.05 \\
\hline 6 & 502.83 & 494.33 & 497.50 & $494.8,8$ & 58.78 & -1.76 \\
\hline$i$ & 523.33 & 520.00 & 514.33 & $52 ! .22$ & 37.39 & 3.46 \\
\hline 8 & 494.93 & 420.50 & $48 n .17$ & 487.17 & 54.05 & -3.30 \\
\hline 9 & 514.17 & $510.2 \%$ & 50 h. 13 & 510.39 & 13.51 & 1.31 \\
\hline 10 & $5: 5.07$ & 513.33 & 513.03 & 514.23 & 1.52 & 2.09 \\
\hline
\end{tabular}

$\bar{x}_{\wedge}=503,77$

$s_{\Lambda}^{2}=56,88$

\begin{tabular}{|c|c|c|}
\hline DESV. TIP. EN UN LAB. $\left(\sigma_{A}\right)$ & - 7,54 & (Repetib.) \\
\hline DESV. TIP. ENTRE LABS $\left(\sigma_{E L}\right)$ & $=16,37$ & (Reproduclb.) \\
\hline COEF. VAR. EN UN LAB., \% $\left(V_{A} \%\right)$ & $=1,50$ & (Repefib.) \\
\hline OEF VAR. ENTRE LABS.,\% $\left(V_{E L} \%\right.$ & 3,25 & (Reproducib.) \\
\hline
\end{tabular}

